\title{
ON THE EXTREME POINTS OF SOME SETS OF ANALYTIC FUNCTIONS ${ }^{1}$
}

\author{
JOHN G. MILCETICH
}

\begin{abstract}
Let $A$ denote the set of analytic functions defined on the open unit disc. The extreme points of $F=\{f \in A: f(0)=0$ and $|\operatorname{Re} f(z)|<\pi / 2\}$ are determined. Also a partial characterization is given for the extreme points of $G_{a}=\{f \in A: f(0)=1$ and $|\arg f(z)|<a \pi, 2\}, 0$ $<\alpha<1$.
\end{abstract}

Introduction. The set $A$ of analytic functions defined on the open unit disc $U$ with the topology of uniform convergence on compact subsets of $U$ is a locally convex linear topological space. Recall that $f \in F \subset A$ is called an extreme point of $F$ if $f$ cannot be written as a proper convex combination of two distinct points of $F$. If $F$ is the set of $f \in A$ such that $f(0)=1$ and $\operatorname{Re} f(z)>0$, the classical Herglotz formula shows that the extreme points of $F$ are precisely the functions $f(z)=\left(e^{i \theta}+z\right)\left(e^{i \theta}-z\right)^{-1}$. When $F$ is the set of $f \in A$ such that $|f(z)| \leq 1$, it is known that $f$ is an extreme point of $F$ if and only if $f \in F$ and

$$
\int_{-\pi}^{\pi} \log \left[1-\left|f\left(e^{i \theta}\right)\right|\right] d \theta=-\infty \quad[4, \mathrm{p} .125]
$$

Lately there has been considerable interest in finding the extreme points of certain other subsets of $A[1],[2],[3],[5],[6],[7]$.

In this paper we characterize the extreme points of

$$
F=\{f \in A: f(0)=0 \text { and }|\operatorname{Re} f(z)|<\pi / 2\} \text {. }
$$

We also give a partial characterization for the extreme points of

$$
G_{a}=\left\{f \in A: f(0)=1,|\arg f(z)|<\alpha_{\pi} / 2\right\}
$$

where $0<a<1$.

Received by the editors April 18, 1973 and, in revised form, September 8, 1973. AMS (MOS) subject classifications (1970)。 Primary 30A32; Secondary 30A76, $30 \mathrm{A78}$.

Key words and phrases. Extreme points, subordination, $H^{p}$ spaces.

1 The author wishes to thank Professor W. E. Kirwan for suggesting this subject for investigation. 
D. J. Hallenbeck and T. H. MacGregor [5] have shown that if $f \in H^{p}$, $p>1$, and if $H$ denotes the set of functions subordinate to $f$, then all functions of the form $f \circ \phi$ are extreme points of $H$, where $\phi(0)=0$ and $\phi$ is an inner function. Their proof depends on a theorem due to Ryff [8]. Part of our results follows from the result of Hallenbeck and Mac Gregor, but we have a direct argument that avoids the use of their result.

Every function in $F$ or $G_{\alpha}, 0<\alpha<1$, lies in $H^{1}$; in fact, $F \subset H^{p}$ for all $p<\infty$ and $G_{\alpha} \subset H^{p}$ for all $p<1 / \alpha[4$, pp. 10, 13, 56]. Consequently these functions possess boundary values almost everywhere and can be represented by means of a Poisson integral.

1. Let $S=\{w:|\operatorname{Re} w|<\pi / 2\}$ and let $F$ denote the set of analytic functions mapping $U$ into $S$ with $f(0)=0$. For a function $f \in F$, the boundary values $f(\theta)=\lim _{r \rightarrow 1} f\left(r e^{i \theta}\right)$ exist a.e. on $[-\pi, \pi]$. Let $E_{f}=\{\theta: d(f(\theta), \partial S)>$ $0\}$, where $\partial S$ denotes the boundary of $S$ and $d(f(\theta), \partial S)$ denotes the distance from $f(\theta)$ to $\partial S$.

Theorem 1. $f$ is an extreme point of $F$ if and only if $f \in F$ and the measure of $E_{f}$ is zero.

Proof. Let $f \in F$ and suppose $E_{f}$ has measure zero. Let $h$ be analytic in $U$ with $h(0)=0$ such that $f \pm h \in F$. In order to show that $f$ is an extreme point of $F$, it is sufficient to show that $h \equiv 0$. Set

$$
g_{1}(z)=\exp (f(z)+h(z)) \text { and } g_{2}(z)=\exp (f(z)-h(z)) \text {. }
$$

Then $g_{1}$ and $g_{2}$ map $U$ into $\left\{w: e^{-\pi / 2}<|w|<e^{\pi / 2}\right\}$. Almost everywhere on $[-\pi, \pi], e^{-\pi / 2} \leq\left|g_{i}(\theta)\right| \leq e^{\pi / 2}$ for $i=1,2$. Set $E_{1}=\{\theta:|\exp f(\theta)|=$ $\left.e^{-\pi / 2}\right\}$ and $E_{2}=\left\{\theta:|\exp f(\theta)|=e^{\pi / 2}\right\}$. Since the boundary values of $f$ and of $f \pm b$ exist a.e., it follows that the boundary values of $h$ exist a.e. If $\theta \in E_{1}$, then $|\exp h(\theta)| \geq 1$ and $|\exp (-h(\theta))| \geq 1$ a.e. because $e^{-\pi / 2} \leq$ $\left|g_{i}(\theta)\right|$ a.e. for $i=1,2$. Hence $|\exp h(\theta)|=1$ a.e. on $E_{1}$. Similarly $|\exp h(\theta)|=1$ a.e. on $E_{2}$. But, by hypothesis, $E_{1} \cup E_{2}$ has measure $2 \pi$ and since $\exp h(z)$ is a bounded function it follows that

$$
\sup _{|z|<1}|\exp h(z)|=\text { ess } \sup |\exp h(\theta)|=1 .
$$

But $\exp h(0)=1$, so that $\exp h(z) \equiv 1$ by the maximum modulus theorem. Therefore $h(z) \equiv 0$ and $f$ is an extreme point of $F$.

Conversely, if $E_{f}$ has positive measure, there exists $\epsilon, 0<\epsilon<1$, such that $H=\{\theta: d(f(\theta), \partial S)>\epsilon\}$ has positive measure. Let $E_{1}, E_{2}$ be 
disjoint closed subsets of $H$ of positive measure. Let $\omega_{i}(z)$ be the harmonic measure of $E_{i}$ at $z$. Then $0<\omega_{i}(z)<1$ and if $\theta \in E_{i}, \omega_{i}(\theta)=1$ a.e.; if $\theta \notin E_{i}, \omega_{i}(\theta)=0$. Set

$$
u(z)=\epsilon\left\{\omega_{1}(z) / m_{1}-\omega_{2}(z) / m_{2}\right\} \min \left\{m_{1}, m_{2}\right\}
$$

where $m_{i}$ is the measure of $E_{i}$. Then $|u(\theta)| \leq \epsilon$ a.e. and if $\theta \notin E_{1} \cup E_{2}$, $u(\theta)=0$. If $v(z)$ is the harmonic conjugate of $u(z)$ which vanishes at $z=0$ and if $h(z)=u(z)+i v(z)$,

$$
\begin{aligned}
h(0) & =\frac{1}{2 \pi} \int_{-\pi}^{\pi} u(\theta) d \theta \\
& =\frac{\epsilon}{2 \pi} \cdot \min \left\{m_{1}, m_{2}\right\} \cdot\left\{\int_{E_{1}} \frac{\omega_{1}(\theta)}{m_{1}} d \theta-\int_{E_{2}} \frac{\omega_{2}(\theta)}{m_{2}} d \theta\right\}=0 .
\end{aligned}
$$

If $\theta \in E_{1} \cup E_{2}, d(f(\theta), \partial S)>\epsilon$ and $|\operatorname{Re} h(\theta)| \leq \epsilon$ a.e. on $E_{1} \cup E_{2}$, so that $|\operatorname{Re}\{f(\theta) \pm h(\theta)\}| \leq \pi / 2$ a.e. on $E_{1} \cup E_{2}$. If $\theta \notin E_{1} \cup E_{2}$, then $\operatorname{Re} h(\theta)=0$ and $|\operatorname{Re}\{f(\theta) \pm h(\theta)\}| \leq \pi / 2$ a.e. But

$$
|\operatorname{Re}\{f(z) \pm h(z)\}| \leq \frac{1}{2 \pi} \int_{-\pi}^{\pi} P_{r}(\theta-t)|\operatorname{Re}\{f(t) \pm h(t)\}| d t \leq \frac{\pi}{2}
$$

where $z=r e^{i \theta}$ and $P_{r}(\theta-t)$ is the Poisson kernel. Therefore $f \pm h \in F$ and $f$ is not an extreme point of $F$ since $h \neq 0$.

2. Let $W_{a}=\left\{z:|\arg z|<\alpha_{\pi} / 2\right\}$ for $0<\alpha<1$ and let $G_{\alpha}$ denote the set of analytic functions with normalization $f(0)=1$ which map $U$ into $W_{a}$. We need the following lemma before discussing the extreme points of $G_{a}$.

Lemma. If $z_{0} \in W_{a}$, then

$$
\sup \left\{|w|: z_{0} \pm w \in W_{a}\right\} \leq\left|z_{0}\right| \max \left\{1,2 \tan \left(\alpha_{\pi} / 2\right)\right\} .
$$

Proof. For each $\theta, 0 \leq \theta<\pi$, let $1_{\theta}$ be the line through $z_{0}$ which makes the angle $\theta$ with the positive real axis. If $\arg w=\theta=\arg z_{0}$, then $z_{0} \pm w \in W_{a}$ if and only if $|w|<\left|z_{0}\right|$. If $\theta=\arg w, \theta \neq \arg z_{0}$, let $z_{1}(\theta)$ be the intersection of $1_{\theta}$ with

$$
\bar{W}_{a} \cap\left\{z: \operatorname{Re} z \leq \operatorname{Re} z_{0}\right\}
$$

and let $z_{2}(\theta)$ be the intersection of $1_{\theta}$ with

$$
\bar{W}_{\alpha} \cap\left\{z: \operatorname{Re} z \geq \operatorname{Re} z_{0}\right\} \text {. }
$$

Here $\bar{W}_{\alpha}$ denotes the closure of $W_{\alpha} \cdot$ (For $\theta=\pi / 2$, choose either point for 
$z_{1}(\theta)$, the other for $z_{2}(\theta)$.) Then $z_{0} \pm w \in W_{a}$ if and only if

$$
|w|<\min \left\{\left|z_{0}-z_{1}(\theta)\right|,\left|z_{0}-z_{2}(\theta)\right|\right\} \leq\left|z_{0}-z_{1}(\theta)\right| \text {. }
$$

Thus

$$
\begin{aligned}
\sup \left\{|w|: z_{0} \pm w \in W_{a}\right\} & \leq \sup \left\{\left|z_{0}-z\right|: z \in \partial W_{a}, \operatorname{Re} z \leq \operatorname{Re} z_{0}\right\} \\
& =\max \left\{\left|z_{0}\right|,\left|z_{0}-z_{1}(\pi / 2)\right|,\left|z_{0}-z_{2}(\pi / 2)\right|\right\} .
\end{aligned}
$$

But it is easily seen that $\left|z_{0}-z_{i}(\pi / 2)\right| \leq 2\left|z_{0}\right| \tan \left(\alpha_{\pi} / 2\right)$ and the lemma follows.

Theorem 2. Let $f \in G_{\alpha}$ and $E_{f}=\left\{\theta: d\left(f(\theta), \partial W_{\alpha}\right)>0\right\}$. If $E_{f}$ has measure zero, then $f$ is an extreme point of $G_{a}$.

Proof. Suppose $h$ is analytic on $U, h(0)=0$, and $f \pm h \in G_{a}$. By the lemma, $|h(z)| \leq M|f(z)|$ where $M=\max \{1,2 \tan (\alpha \pi / 2)\}$ and hence $h(z) / f(z)$ is a bounded analytic function. Suppose $h(z)$ is not identically zero. Since $f(\theta) \in \partial W_{a}$ a.e. and $f(\theta) \pm h(\theta) \in \bar{W}_{a}$ a.e., $\arg h(\theta)=\arg f(\theta)+k(\theta) \pi$ a.e. where $k(\theta) \in \mathbf{Z}$. Since $f(\theta)$ is nonzero a.e., $h(\theta) / f(\theta)$ is defined a.e. and the above shows that $h(\theta) / f(\theta)$ is real a.e. Since $h(z) / f(z)$ is a bounded function with real boundary values a.e., $h(z) / f(z)$ is everywhere real and is therefore a constant. Since $h(0)=0$, this constant is zero and $h$ is identically zero. Therefore $f$ is an extreme point of $F$.

The method used in the above proof easily yields the following generalization.

Let $K$ be a convex polygon (not necessarily compact) which is contained in some wedge $W_{a}, 0<a<1$. Let $F$ denote the set of analytic functions mapping $U$ into $K$ and normalized such that $f(0)=z_{0}$, where $z_{0}$ is an interior point of $K$. If $f \in F$ and $\{\theta: d(f(\theta), \partial K)>0\}$ has measure zero, then $f$ is an extreme point of $F$.

In the direction of the converse of Theorem 2 we can only give a partial result. However this does not depend on the fact that $0<a<1$.

Theorem 3. Let $f \in G_{a}$ and suppose there is an interval $I=\{\theta: y<\theta$ $<\beta\}$ with the property that $d\left(f(\theta), \partial W_{a}\right)>\epsilon>0$ a.e. on I and ess $\sup _{\mathrm{P}}|f(\theta)|$ $\leq M$. Then $f$ is not an extreme point of $G_{\alpha}$.

Proof. For $\beta \leq \theta \leq 2 \pi+\gamma$, define $u(\theta)=-\theta$ and for $\gamma \leq \theta \leq \beta$, let $u(\theta)$ be a $C^{1}$ function with $u(\beta)=-\beta, u(\gamma)=-2 \pi-\gamma$ and $u^{\prime}(\beta)=u^{\prime}(\gamma)=-1$. Then $u$ is continuously differentiable on $[\gamma, 2 \pi+\gamma]$. For $z=r e^{i \theta}$, set 


$$
u(z)=\frac{1}{2 \pi} \int_{\gamma}^{2 \pi+\gamma} P_{r}(\theta-t) u(t) d t
$$

and let $v(z)$ be the harmonic conjugate of $u$ vanishing at $z=0$. Then $u$ and $v$ are both continuously extendible to $|z| \leq 1[4$, p. 83]. Set

$$
h_{1}(z)=B^{-1} \exp \{i u(z)-v(z)\}
$$

where $B=\max |z|_{\leq 1}|\exp \{i u(z)-v(z)\}|$ and set

$$
h(z)=\left(\epsilon_{0} /(M+1)\right) \cdot z f(z) \cdot h_{1}(z)
$$

where $\epsilon_{0}=\min \{1, \epsilon\}$. Almost everywhere on $I, d\left(f(\theta), \partial W_{a}\right)>\epsilon \cdot$ and $|h(\theta)|$ $<\epsilon$, so $f(\theta) \pm h(\theta) \in \bar{W}_{a}$ : For $\theta \notin l$, arg $h(\theta)=\arg f(\theta)$ a.e., and $|h(\theta)| \leq$ $|f(\theta)|$ a.e., so $f(\theta) \pm h(\theta) \in \bar{W}_{a}$. The Poisson integral representation shows that $\operatorname{Re}\{f(z) \pm h(z)\}>0$ so that a single-valued branch of $\arg \{f(z) \pm b(z)\}$ can be chosen. Choosing the branch so that arg $1=0$ and using the Poisson representation, it then follows that

$$
|\arg f(z) \pm h(z)|<\alpha_{\pi} / 2 \text { for } z \in U \text {. }
$$

Hence $f \pm h \in G_{a}$ and $f$ is not an extreme point of $G_{a}$.

In [1], D. A. Brannan, J. G. Clunie, and W. E. Kirwan have shown that the extreme points of $G_{\alpha}, \alpha>1$, are precisely the functions

$$
f_{x}(z)=((1+x z) /(1-x z))^{\alpha}, \quad|x|=1 .
$$

When $0<\alpha<1$, these functions are extreme points of $G_{\alpha}$ but they do not exhaust the set of extreme points as Theorem 2 shows.

\section{REF ERENCES}

1. D. A. Brannan, J. G. Clunie and W. E. Kirwan, On the coefficient problem for functions of bounded boundary rotation, Ann. Acad. Sci. Fenn. AI, no. 523 (1973), 18 pp.

2. L. Brickman, D. J. Hallenbeck, T. H. Mac Gregor and D. R. Wilken, Convex hulls and extreme points of families of starlike and convex mappings, Trans. Amer. Math. Soc. 185 (1973), 413-428.

3. L. Brickman, T. H. Mac Gregor and D. R. Wilken, Convex hulls of some classical families of univalent functions, Trans. Amer. Math. Soc. 156 (1971), 91-107. MR 43 \#494.

4. P. L. Duren, Theory of $H^{p}$ spaces, Pure and Appl. Math., vol. 38, Academic Press, New York, 1970. MR 42 \#3552. 
5. D. J. Hallenbeck and T. H. MacGregor, Subordination and extreme point theory (to appear).

6. H. M. Hilden, A characterization of the extreme points of some convex sets of analytic functions, Duke Math. J. 37 (1970), 715-723. MR 42 \#6618.

7. T. H. MacGregor, Applications of extreme point theory to univalent functions, Michigan Math. J. 19 (1972), 361-376.

8. J. V. Ryff, Subordinate $H^{p}$ functions, Duke Math. J. 33 (1966), 347-354. MR $33 \# 289$.

DEPARTMENT OF MATHEMATICS, FEDERAL CITY COLLEGE, WASHINGTON, D. C. 20001 